\title{
Determination of heat release rate disturbances in unconfined flames based on fluctuations in the travel time of ultrasonic waves
}

\author{
J.Lia ${ }^{\mathrm{a}, \mathrm{b}}$, F.Richecoeur ${ }^{\mathrm{a}, \mathrm{b}}$, T.Schuller ${ }^{\mathrm{a}, \mathrm{b}, *}$
}

${ }^{a}$ CNRS, UPR 288 Laboratoire d'Energétique Moléculaire et Macroscopique, Combustion (EM2C), Grande Voie des Vignes, 92295 Châtenay-Malabry, France.

${ }^{b}$ Ecole Centrale Paris, Grande Voie des Vignes, 92295 Châtenay-Malabry, France.

\begin{abstract}
The paper presents a new method to measure heat release rate disturbances from flames when optical access is limited. The technique is based on the determination of the travel time of ultrasonic waves propagating through the flow. The link between heat release rate and sound travel time disturbances depends on the configuration considered. An expression is established here for the case of unconfined premixed flames driven by buoyancy forces associated with the Kelvin-Helmholtz instability formed by the interaction between accelerating hot burned gases and cold ambient air at rest. The system and the principle used for the determination of the sound travel time are then presented and validated under non-reacting conditions. Effects of the main parameters on the precision of this detection technique are examined experimentally. Measurements in reacting conditions are compared to heat release rate data obtained with another technique based on the chemiluminescence
\end{abstract}

\footnotetext{
*Corresponding author.

Email address: thierry.schuller@ecp.fr (T.Schuller)
} 
emission. A good agreement is obtained between both signals for the different cases explored demonstrating the sensitivity of the proposed technique.

Keywords: Heat release rate; Chirp excitation method; Ultrasonic waves; Flame flickering; Diagnostic.

\section{Introduction}

Monitoring and controlling heat release rate disturbances is an impor3 tant issue in practical combustion chambers because these perturbations are 4 the sources of unsteady thermal stress, direct and indirect combustion noise 5 when entropy waves are accelerated by the mean flow (see for example the 6 recent review by Candel et al. (2009)). They may promote self-sustained 7 thermoacoustic instabilities causing potential severe damages and early ag8 ing of components of the combustion chamber (Candel 2002; Lieuwen and 9 Yang 2005). It is therefore necessary to have reliable tools to estimate these erally rely on optical diagnostics.

12

A widely used technique to estimate heat release rate is to collect the chemiluminescence emission from the flame. Chemiluminescence from hydrocarbon flames results from naturally excited intermediate radicals, such as $\mathrm{OH}^{*}, \mathrm{CH}^{*}$ and $\mathrm{C}_{2}^{*}$, formed within the flame front and emitting a photon during the transition to a lower energy state. These radicals are often considered as good markers of heat release rate (Gaydon 1957). Recording the natural emission from the flame is the simplest technique to obtain time-resolved data and is often used to estimate heat release rate perturba- 
tions by collecting the total emission from the flame (see for example Hurle et al. (1968); Price et al. (1969); Ducruix et al. (2000)) or by local point measurements (Kojima et al. 2000; Hardalupas and Orain 2004). This has for example been validated in laminar premixed flames submitted to flow modulations in the absence of mixture composition inhomogeneities, where fluctuations in the chemiluminescence emission were shown to be proportional to flame surface area disturbances (Schuller et al. 2002). Spectral selection of $\mathrm{OH}^{*}$ or $\mathrm{CH}^{*}$ emissions is generally preferred for hydrocarbon flames but it is also possible to use other chemiluminescent species, such as $\mathrm{C}_{2}^{*}$ or $\mathrm{CO}_{2}^{*}$ (Samaniengo et al. 1995; Najm et al. 1998; Docquier et al. 2000). In more complex configurations, different additional phenomena modify this relation and effects of the turbulence intensity (Ayoola et al. 2006; Lauer and Sattelmayer 2010), flame strain rate (Hardalupas and Orain 2004; Ayoola et al. 2006; Nori and Seitzman 2009), flame front curvature (Najm et al. 1998), local mixture composition (Hardalupas and Orain 2004), temperature and pressure (Docquier et al. 2000; Higgins et al. 2001; Ikeda et al. 2002) must be included to obtain quantitative estimates of the heat release rate. Measurements are then often limited to flame images for qualitative analysis except in a few studies where the signal is calibrated using specific post-processing procedures (see for example Lauer et al. (2011); Palies et al. (2010)). One problem is that the chemiluminescence emission yields an information integrated in the line-of-sight and it is difficult to obtain spatially resolved data. The signal is also more difficult to interpret in non perfectly premixed systems (Balachandran et al. 2005b; Kim et al. 2010). 
One possibility to improve spatial resolution is to use Laser Induced Fluorescence (LIF) by stimulating certain electronic transitions from the radicals present in the flow with a laser sheet intersecting the flame front. OH LIF is widely used since the $\mathrm{OH}$ peak mole fraction occurs in the burned gases just behind the reaction zone and the gradients of the fluorescence signal are found to correlate well with the position of the local flame front in turbulent flames (see for example Knikker et al. (2002); Sadanandan et al. (2008)). The $\mathrm{CH}$ fluorescence signal is a better tracer of the reaction zone due to its relatively short lifetime and its abundance over a narrow region in the flame zone (Nguyen and Paul 1996; Donbar et al. 2000), even though large deviations with heat release rate were highlighted for diluted flames during transient phenomena (Vagelopoulos and Frank 2005). Time resolved data are more difficult to obtain due to the limited repetition rates and energy delivered per pulse from the lasers, although $\mathrm{OH}$ and $\mathrm{CH}$ planar measurements at a few $\mathrm{kHz}$ were recently reported to characterize transient phenomena in complex turbulent reacting flows (see for example Tanahashi et al. (2008); Boxx et al. (2009); Stöhr et al. (2011)).

While these techniques enable to locate the flame front, quantitative estimates of spatially resolved data for the heat release rate are still challenging. It is generally admitted that the intermediate radical $\mathrm{HCO}$ present in the flame front correlates well with heat release rate but the stimulation of an electronic transition with a detectable fluorescence signal raises several difficulties (Jeffries et al. 1991). It was shown that the concentration of HCO can however be estimated by the product of the $\mathrm{OH}$ and $\mathrm{CH}_{2} \mathrm{O}$ radical concen- 
trations formed around the flame, whose fluorescences are easier to obtain. This has motivated a series of studies based on LIF measurements at different excitation wavelengths to estimate the local distribution of heat release rate in flow configurations of increasing complexity (Najm et al. 1998; Paul and Najm 1998; Fayoux et al. 2005; Balachandran et al. 2005a; Ayoola et al. 2006). This type of simultaneous measurements in unsteady flows remain however heavy to implement and require high power well-tuned laser beams at different wavelengths and specific optics.

The methods briefly presented above are all based on optical detection of radicals present in the flame and are difficult to implement when the optical access is limited. The objective of this study is to develop one original solution based on an acoustic method. This new technique relies on determination of the travel time of ultrasonic waves propagating through a flame.

Flames submitted to acoustic disturbances behave as low-pass filters and are only sensitive to the velocity component of sound waves (Ducruix et al. 2003). Low frequency pressure disturbances are transmitted through a flame without distortion. The cut-off frequency of the flame response is reached when the velocity perturbation wavelength is of the order of the flame length $\lambda \sim L$, which is typically limited to about $2 \mathrm{kHz}$ for most typical applications. In the intermediate range of frequencies, sound waves are scattered by the strong jump in density across the flame (Cho 2009) and by turbulent wrinkles which develop along the flame for frequencies ranging from about 1 to $20 \mathrm{kHz}$ (Lieuwen 2001). The incoherent scattered sound power first 
increases exponentially with frequency and then saturates to a certain level when compared to the coherent sound power incident on the flame (Lieuwen et al. 2002). When the wavelength is of the order of the flame thickness, pressure perturbations may then strongly interact with the preheat zone $\lambda \sim \delta$ and consequently modify the local reaction rate (Peters and Ludford 1983; McIntosh 1991; Ledder and Kapila 1991). This occurs only at very high frequencies of the order of $100 \mathrm{kHz}$ for typical hydrocarbon-air flames, except when flames are submitted to high accelerations at very high forcing levels (Durox et al. 1997a; Durox et al. 1998; Wangher et al. 2008).

Information carried by the scattered field from ultrasonic waves impinging the flow has already been used to analyze vorticity production in turbulent jets and swirling jets (Baerg and Schwarz 1966; Fabrikant 1983; Oljaca et al. 1998; Poulain et al. 2004) as well as to detect temperature fluctuations in inert turbulent flows (Contreras and Lund 1990; Elicer-Cortes et al. 2004). In the case of reacting flows, these investigations were limited yet to the examination of the scattered sound field by the flame as a function of the geometric properties of the reaction front and the characteristics of turbulence (Lieuwen et al. 2002; Richecoeur et al. 2009). It is shown in these references that flame front wrinkles induced by turbulence result in Doppler shifted scattered waves, the coherent scattered signal being significantly damped compared to the incident coherent ultrasonic pressure disturbances, but there has been no attempt yet to use ultrasonic waves to probe reacting flow properties.

This paper focuses on the use of ultrasonic waves and considers distur- 
bances of the sound travel time $\Delta t^{\prime}$ between an emitter and a receiver as an indicator of perturbations in the flame width. This information is then used to reconstruct low frequency heat release rate disturbances $\dot{Q}^{\prime}$ for unconfined premixed flames driven by buoyancy induced oscillations. This phenomenon called "flickering" is associated with the Kelvin-Helmholtz instability formed by the buoyant interaction between the hot burned gases and cold ambient air (Durox et al. 1997b). It produces small heat release perturbations with typical frequencies around $10 \mathrm{~Hz}$ at ground level gravity (Durox et al. 1990; Kostiuk and Cheng 1995) and provides a generic configuration to validate our acoustic methodology for heat release rate reconstruction in the absence of turbulence and external forcing device. Analysis of turbulence effects is out of the scope of the present study and this important topic will be the object of future investigations. Reconstruction of heat release rate for flames submitted to harmonic flowrate modulation has already been undertaken ( $\mathrm{Li}$ et al. 2011) and the present investigation constitutes the second validation of the technique when gravity effects must be considered.

The link between the rate of change of the sound travel time $\mathrm{d} \Delta \mathrm{t} / \mathrm{dt}$ for ultrasonic waves crossing the flame and heat release rate fluctuations $\dot{Q}^{\prime}$ is established in section 2. The experimental configuration is detailed in section 3 , while the methodology developed to determine the travel time of ultrasonic waves together with the post-processing of the incident and transmitted signals are presented in section 4 . This relies on a pulse compression technology used for radar detection. The choice of the type of forcing signal to probe the flow is discussed in section 5. Validation of the acoustic methodology in 
Consider the case of a plane sound wave traveling through a homogeneous gas over a distance $L$ at fixed temperature and composition characterized by 
a speed of sound $c_{u}$. The propagation time $\Delta t_{0}$ is given by:

$$
\Delta t_{0}=\frac{L}{c_{u}}
$$

The burned gases around a flame modify the speed of sound along the acoustic path and consequently change also the sound propagation time. This configuration is represented in Fig. 1 where acoustic waves emitted by a tweeter propagate through three different media corresponding to ambient air, the burned gases and the fresh reactive mixture. In the analysis developed below, ambient air and the unburned gases are characterized by the same speed of sound $c_{u}$. This approximation is reasonable for air-combustion systems due to the strong dilution by nitrogen when the reactants are injected at ambient temperature. This situation can be schematically represented by two different media represented in the right part in Fig. 1. The first medium is composed of burned gases characterized by a speed of sound $c_{b}$ over a length of $L_{f}$ and the second is filled with ambient air characterized by a speed of sound $c_{u}$ and a length $L-L_{f}$. In this case, Eq. (1) is modified and the propagation time $\Delta t$ of sound waves from the emitter to the receiver now writes:

$$
\Delta t=\frac{L-L_{f}}{c_{u}}+\int_{0}^{L_{f}} \frac{\mathrm{dx}}{c_{b}}
$$

By introducing in this expression the sound travel time in the absence of flame $\Delta t_{0}$ which remains constant for a fixed emitter to receiver distance 
and taking first order perturbations one obtains:

$$
\Delta t^{\prime}=-\frac{L_{f}^{\prime}}{c^{*}}
$$

The link between fluctuations in the sound travel time $\Delta t^{\prime}$ and perturbations in the heat release rate $\dot{Q}^{\prime}$ must now be established. The main steps of the derivation are presented below by assuming that the volume of the 
burned gases $V_{b}$ depends on the flame surface area $A_{f}$ of the conical flame:

$$
V_{b}=\alpha L_{f} A_{f}
$$

where $L_{f}$ is the apparent dimension of the burned gases in the line of sight of the emitter and receiver and $\alpha$ denotes a constant coefficient for fixed flow operating conditions and experimental arrangement. Since the flame is anchored at the burner rim, a mass balance between the burner outlet and the flame front yields $v_{u} A=S_{L} A_{f}$, where $S_{L}$ denotes the laminar burning velocity, $v_{u}$ represents the flow velocity at the burner outlet and $A$ indicates the section area of the burner outlet. This yields an expression for the hot plume width $L_{f}$ as a function of the burned gases volume:

$$
L_{f}=\frac{S_{L}}{\alpha v_{u} A} V_{b}
$$

In this expression, $S_{L}$ only depends on the equivalence ratio, $v_{u}$ is kept constant and $\alpha$ is time independent so that $L_{f}$ can be substituted in Eq. (3) to yield:

$$
\Delta t^{\prime}=-\frac{S_{L}}{\alpha v_{u} A c^{*}} V_{b}^{\prime}
$$

[Figure 2 about here.]

For buoyancy driven oscillations, the flow can be considered isobaric. Using the simplified model presented in Fig. 2, a global energy balance over 
the control volume yields:

$$
\frac{\gamma p}{\gamma-1}\left(\frac{\partial V_{u}}{\partial t}+\frac{\partial V_{b}}{\partial t}\right)=\dot{m}_{u} c_{p} T_{u}-\dot{m}_{b} c_{p} T_{b}+\dot{Q}
$$

where the lateral surface of the control volume in contact with ambient air is supposed impermeable $\left(\dot{m}_{i}=0\right)$ and adiabatic $\left(\dot{Q}_{i}=0\right)$. The ratio of specific heat capacity $\gamma=c_{p} / c_{v}$ and ambient pressure $p$ appear on the left side of Eq. (7), while the rate of heat released in the control volume $\dot{Q}$ and the sensible enthalpy fluxes $\dot{m} c_{p} T$ at the unburned (subscript $u$ ) and burned (subscript $b$ ) limits are responsible for changes in the unburned $V_{u}$ and burned $V_{b}$ volumes. In this expression, $T$ denotes the temperature and $\dot{m}$ represents the mass flow rate. A first order perturbation analysis of Eq. (7) yields at low frequency:

$$
\frac{\gamma p}{\gamma-1} \frac{\partial V_{b}^{\prime}}{\partial t}=\dot{Q}^{\prime}
$$

where fluctuations in the fresh reactants volume were neglected since $V_{u} / V_{b} \approx$ $\rho_{b} / \rho_{u}=T_{u} / T_{b} \ll 1$. For methane-air flames $T_{b} / T_{u} \simeq 5-8$ depending on the mixture equivalence ratio $\phi$.

Combining Eqs. (6) and (8), one finally finds that heat release rate disturbances induced by buoyancy effects are proportional to the rate of change in the sound travel time between the transmitter and receiver:

$$
\dot{Q}^{\prime}=-\frac{\gamma p}{\gamma-1} \frac{\alpha v_{u} A c^{*}}{S_{L}} \frac{\partial \Delta t}{\partial t}
$$

This last relation shows that it is possible to estimate heat release rate perturbations induced by flame flickering from fluctuations in the sound propagation time of ultrasonic waves crossing the hot plume of unconfined flames. 
Heat release rate disturbances are then proportional to the rate of change of the sound travel time $\partial \Delta t / \partial t \propto \dot{Q}^{\prime}$. The remaining part of this paper is devoted to validation of Eq. (9).

\section{Experimental configuration}

[Figure 3 about here.]

Experiments are conducted on an axisymmetric burner with a $20 \mathrm{~mm}$ outlet nozzle diameter. Laminar premixed methane-air flames are anchored at the burner rim. The flow rates of methane and air are premixed before entering the burner and the equivalence ratio can be modified by adjusting air and methane Bronkhorts massflow controllers. Ultrasonic pulses transmitted to air are generated by a small tweeter placed on one side of the setup, with a working bandwidth from 5 to $50 \mathrm{kHz}$. The acoustic waves are recorded by two microphones M1 and M2 (B\&K, type 4938, working bandwidth 4 $\mathrm{Hz}-70 \mathrm{kHz}$ ) before and after their passage through (a) a flame or (b) the corresponding jet flow in the absence of combustion. These microphones are alined so that ultrasonic waves cross on the burner symmetry axis in Fig. 3. A photomultiplier (PM) is also placed on the side of the burner to record the light emission from intermediate radicals present within the flame front. For the laminar premixed flames considered here, it is possible to estimate heat release rate perturbations $\dot{Q}^{\prime}$ by examining fluctuations in the chemiluminescence intensity $I$ of free radicals $\mathrm{OH}^{*}, \mathrm{C}_{2}{ }^{*}$ or $\mathrm{CH}^{*}$ present in the reaction zone (Price et al. 1969; Schuller et al. 2002)

$$
\dot{Q}=k I
$$


The coefficient $k$ is a function of the experimental apparatus and mixture composition. It can be determined by a calibration procedure detailed in section 7 .

\section{Determination of the sound propagation time}

[Figure 4 about here.]

A pulse compression technique is used to determine the propagation time of ultrasonic waves between microphones M1 and M2 (Merrill 2008). Figure 4 presents the block diagram of the transmission and reception systems. A train of pulses, as illustrated in Fig. 5, is synthesized by a wave generator. This signal is amplified and transmitted to space using a tweeter. The ultrasonic waves generated are measured with fixed microphones before and after their passage through the perturbed flow. These sound pressure signals are then amplified, high pass filtered and post-processed to compute the cross-correlation between the incident and transmitted sound waves. Each train of pulses is a chirp signal with a constant amplitude $\beta$ and a linear frequency modulation over a certain frequency bandwidth $B$ and a time duration $\tau$ (Fig. 5). This technique enables to obtain a narrow compressed pulse response for the cross-correlation between the incident and transmitted signals with a main peak corresponding to the sought sound travel time $\Delta t$. Provided that the repetition time $t_{r}$ between two successive chirps is large enough, the time resolution of the technique improves as the compression factor $B \tau$ increases (Klauder et al. 1960; Cook and Bernfield 1993; Merrill 2008). 
Theoretically, the incident and transmitted signals can be expressed as:

$$
\begin{gathered}
S_{i}(t)= \begin{cases}\beta_{i} \sin \left(2 \pi\left[f_{i}+\frac{B}{2 \tau} t\right] t\right) & 0 \leqslant t \leqslant \tau \\
0 & \text { elsewhere }\end{cases} \\
S_{t}(t)= \begin{cases}\beta_{t} \sin \left(2 \pi\left[f_{i}+\frac{B}{2 \tau}(t-\Delta t)\right](t-\Delta t)\right) & \Delta t \leqslant t \leqslant \tau+\Delta t \\
0 & \text { elsewhere }\end{cases}
\end{gathered}
$$

The correlation between these two signals is used to obtain a narrow compressed pulse response (Mahafza and Elsherbeni 2004):

$$
R_{\text {corr }}(t)=\frac{1}{\tau} \int_{-\infty}^{+\infty} S_{t}^{*}\left(t^{\prime}\right) S_{i}\left(t^{\prime}-t\right) \mathrm{dt}^{\prime}
$$

The sound propagation time $\Delta t$ is determined by finding the maximum value of the envelope of the cross-correlation response. Examples of ideal generated (Eq. (11)) and transmitted (Eq. (12)) chirps together with the result of the cross-correlation are illustrated in Fig. 6.

[Figure 6 about here.]

Due to the different mechanical and electrical devices in the signal generation and reception, it is not desirable to operate with chirps with a square envelope because of the sudden changes in the signal amplitude at the leading and trailing edges of the sequence. A Hamming window is then applied to the rectangular chirp produced by the signal synthesizer to obtain a smooth transition in amplitude at the beginning and end of the chirp, but the response measured by the different sensors is still distorted as shown in Fig. 7 in the absence of flow between the emitter and receiver. 
[Figure 7 about here.]

[Figure 8 about here.]

To improve results, a pre-processing applied to these raw signals is necessary. The technique is the same for the incident and transmitted signals and will only be detailed for the reference microphone M1 in Fig. 8. The raw signal is first filtered with a high pass zero-phase shift filter to eliminate the low frequency background noise induced by the flow and the generation and measurement devices. The filtered result is shown in Fig. 8(a). A digital Butterworth filter with a cut-off frequency $f=100 \mathrm{~Hz}$ featuring an attenuation of less than $3 \mathrm{~dB}$ in the pass-band and at least $30 \mathrm{~dB}$ in the stop-band was used (Oppenheim and Schafer 1975). This filtered signal and the signal generated by the synthesizer are processed by the cross-correlation algorithm to locate the leading edge of the chirp. The resulting signal is multiplied by a rectangular box function of duration $\tau$ to eliminate the signal outside the chirp as shown in Fig. 8(b). A Hilbert transform is then used to obtain the envelope of the signal, shown in Fig. 8(c). This envelope enables to renormalize the signal presented in Fig. 8(b) and obtain a constant amplitude signal as shown in Fig. 8(d). The pre-processed signals from microphones M1 and M2 are then cross-correlated. The output is again processed by the Hilbert transform to keep only the envelope. The location of the peak of the main lobe yields the propagation time $\Delta t$.

[Figure 9 about here.]

Figure 9 shows an example of the normalized cross-correlation obtained with and without pre-processing. It can be noted that the level reached by the 
side lobes for the pre-processed response is significantly reduced compared to the result obtained without pre-processing. The resolution of the technique is indicated by the width of the main peak calculated at the level 0.5. The pre-processing clearly improves the result.

\section{Parameters optimization for the chirp generation}

[Table 1 about here.]

Different tests were conducted in the absence of flow and flame to optimize the technique for the generation and detection of the chirp ultrasonic signals. The influence of the frequency bandwidth $B$, initial frequency $f_{i}$, chirp duration $\tau$ and chirp repetition rate $t_{r}$ on the precision of the measured sound travel time $\Delta t$ was analyzed in this section. To avoid mixing different neighbor transmitted signals, the duration between two pulses $t_{r}-\tau$ must be larger than the fluctuations of propagation time $\Delta t$. It was checked in this case that results were independent on the repetition rate $t_{r}$. The range explored for the values of the three remaining parameters is synthesized in Tab. 1. In this table, the carrier frequency $f_{0}=f_{i}+B / 2$ is indicated instead of $f_{i}$ to examine effects of the initial frequency. The influence of each parameter was studied separately by keeping the two others fixed. In these experiments, the signals from the microphones were sampled at a frequency $f_{s}=2^{21} \mathrm{~Hz}$ over a duration $0.2 \mathrm{~s}$ and measurements were repeated 100 times for each case explored to obtain the confidence interval of the samples.

[Figure 10 about here.] 
The measured sound travel times are plotted as a function of the frequency bandwidth $B$, pulse duration $\tau$ and carrier frequency $f_{0}$ respectively in Fig. 10. In these plots, error bars correspond to the maximal deviation over 100 repetitions and the black disks denote the mean value. It can be noted that an increase in the frequency bandwidth $B$ or pulse-width duration $\tau$ greatly improves the precision in Fig. 10(a) and (b), but the choice of the pulse-width duration is limited when time resolved data are needed. For example, to obtain time resolved records with a sampling rate of $500 \mathrm{~Hz}, \tau$ has to be less than $2 \mathrm{~ms}$. Theoretically, the carrier frequency $f_{0}$ or initial frequency $f_{i}$ has no effect on the precision (Merrill 2008). Figure 10(c) shows however that results feature some scatter depending on $f_{0}$. This phenomenon is again induced by the different mechanical and electronic responses of the transmitter and receiver devices. In the remaining experiments, the parameters chosen for the chirp generation were fixed to the optimal values indicated in Tab. 2.

\section{[Table 2 about here.]}

\section{Validation in the absence of flame}

The transmission of sound waves between microphones M1 and M2 under non-reacting conditions was investigated. Experiments were conducted for three different cases, (a) without burner, (b) with the burner between the two microphones but in the absence of flow, and (c) for a non-reacting jet flow (Fig. 3). The Reynolds number based on the flow velocity and nozzle diameter $\mathrm{Re}=2900$ corresponds to a laminar jet with a flow velocity typical of the reacting conditions explored in the next section. Parameters used to 
generate the chirp are listed in Tab. 2. Microphones signals were sampled here at a frequency $f_{s}=2^{21} \mathrm{~Hz}$ during a period of $0.2 \mathrm{~s}$. This duration is long enough to collect 80 transmitted chirps for each record.

[Figure 11 about here.]

By modifying the horizontal position of M2 (Fig. 3), it is possible to determine the evolution of the travel time of ultrasonic waves $\Delta t_{0}$ as a function of the distance $L$ between the two microphones. In these experiments, the horizontal distance between the two microphones was increased by steps of $5 \mathrm{~mm}$ to record the different sets of data. The evolution of the difference $\Delta\left(\Delta t_{0}\right)=\Delta t_{0}(L)-\Delta t_{0}\left(L_{0}\right)$ between measurements of the sound travel time recorded for two distances $L$ and $L_{0}$ between microphones M1 and M2 is plotted in Fig. 11 as a function of the distance $\Delta L=L-L_{0}$, where the reference distance corresponds to $L_{0}=300 \mathrm{~mm}$. The difference in sound travel time $\Delta\left(\Delta t_{0}\right)$ increases linearly with $\Delta L$ for all cases explored. All data collapse on the same line indicating that there is no scattering effects due to the presence of the burner or laminar jet. The slope $1 / c_{0}$ indeed fits with predictions from Eq. (1), where $c_{0}=348 \mathrm{~m} . \mathrm{s}^{-1}$ is the speed of the sound in ambient air. This setup and the methodology developed can now be used to examine perturbations in the sound travel time induced by the presence of the flame between the two microphones.

\section{Validation with flame}

[Figure 12 about here.] 
Laminar premixed conical flames are stabilized on the burner rim. As already discussed in the previous sections, while the flow is steady, the tip of the conical flame features a small regular oscillation called flickering caused by the unstable interface between the burned gases and ambient air (Fig. 12, left image). It is also known that a cylindrical tube on the top of the flame stabilizes the flow of the burned gases and the flickering phenomenon is attenuated (Kizirnis et al. 1984; Linteris and Rafferty 2008). This is illustrated in the right image in Fig. 12. Flickering features typical frequencies around $10-20 \mathrm{~Hz}$ (Kostiuk and Cheng 1994; Durox et al. 1997b). This motion generates in turn small low frequency heat release rate perturbations that can be identified by measuring fluctuations in the light emission intensity $I$ recorded by the PM or by determining fluctuations in the sound travel time $\Delta t$ with the microphones M1 and M2.

[Figure 13 about here.]

Measurements are shown for a lean methane-air flame with a bulk velocity $v_{u}=1.5 \mathrm{~m} \cdot \mathrm{s}^{-1}$ and equivalence ratio $\phi=0.9$ with and without a quartz tube on the top of the flame. Signals are recorded here with the same sampling frequency $f_{s}=2^{11} \mathrm{~Hz}$ but over a longer sampling time $1.5 \mathrm{~s}$ than in the experiments in the absence of combustion. The left graph in Fig. 13 shows that the light intensity $I(t)$ and sound travel time $\Delta t$ both feature a periodic motion. Power spectral analysis based on the data collected with the PM and the microphones show that the main peak in both cases corresponds to the oscillation frequency $f=16 \pm 1 \mathrm{~Hz}$. These measurements are in agreement with the flickering frequency deduced from the correlation of (Kostiuk and 
Cheng 1994):

$$
\frac{f^{2} D}{g \xi(\xi-1)}=0.00028\left(\frac{v_{u} D}{\xi \nu}\right)^{2 / 3}
$$

[Figure 14 about here.]

Fluctuations in the chemiluminescence emission intensity $I$ and disturbances in the sound travel time $\Delta t$ result both from heat release rate perturbations. The link between these quantities is obtained by plotting the evolution of the mean value of the light intensity $\bar{I}$ as a function of the flow velocity $v_{u}$ at the burner outlet. Results are presented in Fig. 14 for four flames featuring different equivalence ratios $\phi=0.85,0.90,0.95$ and 1.00 . The evolution is linear $\bar{I}=\epsilon v_{u}$, where the coefficient $\epsilon$ depends on the equivalence ratio of the combustible mixture. It is then possible to obtain an expression for the coefficient $k$ appearing in Eq. (10) linking the heat release rate $\overline{\dot{Q}}$ and the chemiluminescence intensity $\bar{I}$ :

$$
k=\frac{\overline{\dot{Q}}}{\bar{I}}=\frac{\overline{\dot{Q}}}{\epsilon v_{u}}=\frac{\rho_{u} \Delta q A}{\epsilon}
$$


where $\rho_{u}$ is the unburned mixture density, $A$ the cross section area at the burner outlet and $\Delta q$ the heating value per unit mass of reactive mixture. In this expression, it is assumed that all the mixture is burned. Combining Eqs. (9) and (15), the following relation between perturbations in chemiluminescence intensity $I$ and sound travel time $\Delta t$ can be derived:

$$
\frac{d \Delta t}{d t}=\Xi I^{\prime} \quad \text { where } \quad \Xi=-\frac{(\gamma-1)}{\alpha} \frac{S_{L}}{\epsilon v_{u}} \frac{\Delta q}{c_{u}^{2}} \frac{1}{c^{*}}
$$

For a fixed mixture composition and flow velocity, fluctuations in the flame emission are proportional to the rate of change of the sound travel time of acoustic waves crossing the flame.

[Table 3 about here.]

In this study, conical flames featuring a regular flickering of the tip were found for operating conditions corresponding to equivalence ratios $\phi=[0.85$ 1.50]. It was not possible to stabilize flames leaner than $\phi=0.85$ that blowoff. The rich limit $\phi=1.50$ corresponds to a situation where the conical flame tip opens and the flickering phenomenon disappears. One difficulty with fuel rich operating conditions $(\phi \geq 1)$ is that a diffusion flame surrounds the premixed conical flame stabilized in the center region, where the remaining fuel is burned. In these conditions, techniques based on analysis of the chemiluminescence emission do not reproduce heat release rate disturbances (Lauer and Sattelmayer 2010). This study was thus limited to fuel lean flames $(\phi \leq=1)$. Measurements of $d \Delta t / d t$ and $I^{\prime}$ were conducted for lean premixed methaneair flames featuring different inlet flow velocities ranging from $v_{u}=0.84$ to 
$1.68 \mathrm{~m} . \mathrm{s}^{-1}$ and equivalence ratios $\phi$ varying from 0.85 to 1.00. Parameters fixed for the chirp generation are indicated in Tab. 2. Acoustic measurements were sampled at a frequency equal to $400 \mathrm{~Hz}$ corresponding to a repetition time $t_{r}=2.5 \mathrm{~ms}$. The resulting signal for $\Delta t^{\prime}$ was then filtered by a low-pass zero-phase shift filter with a cut-off frequency equal to $40 \mathrm{~Hz}$. The PM signal was sampled at a frequency of $8192 \mathrm{~Hz}$ during a period of $1.5 \mathrm{~s}$ and is filtered with the same low pass filter. Comparisons between the two measurement techniques are presented in Fig. 15 for the six cases listed in Tab. 3. The opposite signal $-I^{\prime}$ is plotted in this figure because the proportionality coefficient $\Xi$ between $I^{\prime}$ and $d \Delta t^{\prime} / d t$ given by Eq. (16) is negative. Signals were also rescaled in this figure to obtain comparable oscillations levels. The two signals $d \Delta t / d t$ and $-I^{\prime}$ match well in phase and relative amplitude for all cases, clearly demonstrating that the rate of change of the sound travel time of ultrasonic waves is proportional to fluctuations in light intensity emission: $d \Delta t / \mathrm{dt} \propto \mathrm{I}^{\prime}$

[Figure 15 about here.]

[Figure 16 about here.]

Instead of plotting the signals $d \Delta t / d t$ and $I^{\prime}$ as a function of time like in Fig. 15, it is also interesting to plot $d \Delta t / d t$ as a function of $I^{\prime}$ for the different operating conditions explored. One example is presented in Fig. 16 for the case C2 (see Tab. 3). The value of the coefficient $\Xi$ appearing in Eq. (16) can then be determined by a linear regression. These values and the corresponding regression coefficients $r^{2}$ are indicated in Tab. 3 and confirm the linear link between $d \Delta t / d t$ and $I^{\prime}$ for all cases explored. Data determined 
experimentally can also be compared to predictions using values indicated in Tab. 4 to estimate the coefficient $\Xi$ appearing in Eq. (16). Predictions are plotted in Fig. 17 and are compared to measurements for two different equivalence ratios $\phi=0.85$ and $\phi=1.00$ as a function of the nozzle outlet flow velocity $v_{u}$. The distributions of experimental results match well again predictions confirming the validity of Eq. (16) and the possibility to detect heat release rate fluctuations by measuring perturbations in the transmission time of ultrasonic waves crossing flames.

[Table 4 about here.]

[Figure 17 about here.]

\section{Conclusion}

A new technique was presented to detect small heat release rate disturbances in premixed flames submitted to low frequency instabilities driven by buoyancy forces when the optical access is limited. This technique is based on the determination of the travel time of ultrasonic waves transmitted through the flames. An experimental validation of the proposed technique was conducted on a generic configuration with a tweeter to produce the ultrasonic waves and two microphones to record the incident and transmitted signals through different laminar flames. An analytical relation was derived linking sound travel time and heat release rate disturbances. Time resolved measurements obtained with the acoustic methodology were compared with optical data based on records of the chemiluminescence emission from the flames. A good agreement was obtained for the different cases explored demonstrating 
that the transmission of ultrasonic waves can effectively be used to estimate heat release rate disturbances. It was also shown that the proposed technique is very sensitive to small disturbances in heat release rate. These results are encouraging and motivate further development of this technique to investigate heat release rate disturbances in more complex configurations.

\section{Aknowledgement}

Jingxuan Li is supported by a doctoral fellowship from China Scholarship Council, Project 111, Grant No. B08009. The authors wish to thank Dr. Daniel Durox for helpful discussion about buoyancy effects and assistances with color Schlieren technique.

\section{References}

Ayoola, B., R. Balachandran, J. Frank, E. Mastorakos, and C. Kaminski (2006). Spatially resolved heat release rate measurements in turbulent premixed flames. Combust. Flame 144(1-2), 1-16.

Baerg, W. and W. Schwarz (1966). Measurements of the scattering of sound from turbulence. J. Acoust. Soc. Am. 39(6), 1125-1132.

Balachandran, R., B. Ayoola, C. Kaminski, A. Dowling, and E. Mastorakos (2005a). Experimental investigation of the nonlinear response of turbulent premixed flames to imposed inlet velocity oscillations. Combust. Flame 143(1-2), 37-55.

Balachandran, R., A. P. Dowling, and E. Mastorakos (2005b, April). Response of turbulent premixed flames subjected to inlet velocity and 
equivalence ratio perturbations. Louvain-la-Neuve. European Combustion Meeting.

Boxx, I., M. Stöhr, C. Carter, and W. Meier (2009). Sustained multi-kHz flamefront and 3-component velocity-field measurements for the study of turbulent flames. Appl. Phys. B-Lasers Opt. 95(1), 23-29.

Candel, S. (2002). Combustion dynamics and control: Progress and challenges. Proc. Combust. Inst. 29(1), 1-28.

Candel, S., D. Durox, S. Ducruix, A.-L. Birbaud, N. Noiray, and T. Schuller (2009). Flame dynamics and combustion noise: progress and challenges. Int. J. Aeroacoustics 8(1), 1-56.

Cho, J. (2009). Analysis of low-frequency wave scattering by turbulent premixed flame. J. Fluid Mech. 634, 137-164.

Contreras, H. and F. Lund (1990). Ultrasound as a probe of turbulence. II. Temperature inhomogeneities. Phys. Lett. A 149(2-3), 127-130.

Cook, C. E. and M. Bernfield (1993). Radar Signals: An Introduction to Theory and Application. Artech House Publishers, London.

Docquier, N., S. Belhalfaoui, F. Lacas, N. Darabiha, and C. Rolon (2000). Experimental and numerical study of chemiluminescence in methane/air high-pressure flames for active control applications. Proc. Combust. Inst. 28(2), 1765-1774.

Donbar, J., J. Driscoll, and C. Carter (2000). Reaction zone structure in turbulent nonpremixed jet flames-from CH-OH PLIF images. Combust. Flame 122(1-2), 1-19. 
Ducruix, S., D. Durox, and S. Candel (2000). Theoretical and experimental determinations of the transfer function of a laminar premixed flame. Proc. Combust. Inst. 28(1), 765-773.

Ducruix, S., T. Schuller, D. Durox, and S. Candel (2003). Combustion dynamics and instabilities: Elementary coupling and driving mechanisms. J. Propuls. Power 19(5), 722-734.

Durox, D., F. Baillot, P. Scouflaire, and R. Prud'homme (1990). Some effects of gravity on the behaviour of premixed flames. Combust. Flame 82(1), 66-74.

Durox, D., F. Baillot, G. Searby, and L. Boyer (1997a). On the shape of flames under strong acoustic forcing: a mean flow controlled by an oscillating flow. J. Fluid Mech. 350, 295-310.

Durox, D., S. Ducruix, and F. Baillot (1998). Strong acoustic forcing on conical premixed flames. Proc. Combust. Inst. 27(1), 883-889.

Durox, D., T. Yuan, and E. Villermaux (1997b). The effect of buoyancy on flickering in diffusion flames. Combust. Sci. and Tech. 124(1-6), 277294.

Elicer-Cortes, J. C., R. Contreras, D. Boyer, M. Pavageau, and R. H. Hernandez (2004). Temperature spectra from a turbulent thermal plume by ultrasound scattering. Exp. Therm. Fluid Sci. 28(8), 803-813.

Fabrikant, A. (1983). Sound scattering by vortex flows. Sov. Phys. Acoust. 29, 152-154.

Fayoux, A., K. Zäringher, O. Gicquel, and J. Rolon (2005). Experimental and numerical determination of heat release in counterflow premixed 
laminar flames. Proc. Combust. Inst. 30(1), 251-257.

Gaydon, A. G. (1957). The spectroscopy of flames. Wiley, New York.

Hardalupas, Y. and M. Orain (2004). Local measurements of the timedependent heat release rate and equivalence ratio using chemiluminescent emission from a flame. Combust. Flame 139(3), 188-207.

Higgins, B., M. Q. McQuay, F. Lacas, and S. Candel (2001). An experimental study on the effect of pressure and strain rate on $\mathrm{CH}$ chemiluminescence of premixed fuel-lean methane/air flames. Fuel 80(11), $1583-1591$.

Hurle, I. R., R. B. Price, T. M. Sugden, and A. Thomas (1968, March). Sound Emission from Open Turbulent Premixed Flames. Proc. Roy. Soc. London Ser. A 303, 409-427.

Ikeda, Y., J. Kojima, and H. Hashimoto (2002). Local chemiluminescence spectra measurements in a high-pressure laminar methane/air premixed flame. Proc. Combust. Inst. 29(2), 1495-1501.

Jeffries, J. B., D. R. Crosley, I. J. Wysong, and G. P. Smith (1991). Laserinduced fluorescence detection of HCO in a low-pressure flame. Proc. Combust. Inst. 23(1), 1847-1854.

Kim, K., J. Lee, B. Quay, and D. Santavicca (2010). Response of partially premixed flames to acoustic velocity and equivalence ratio perturbations. Combust. Flame 157(9), 1731-1744.

Kizirnis, S. W., R. J. Brecha, B. N. Ganguly, L. P. Goss, , and R. Gupta (1984). Hydroxyl (OH) distributions and temperature profiles in a premixed propane flame obtained by laser deflection techniques. Appl. 
Opt. 23, 3873-3880.

Klauder, J., A. C. Price, S. Darlington, and W. J. Albersheim (1960, July). The theory and design of chirp radars. Bell Sys. Tech. J. 39(4), $745-808$.

Knikker, R., D. Veynante, and C. Meneveau (2002). A priori testing of a similarity model for large eddysimulations of turbulent premixed combustion. Proc. Combust. Inst. 29(2), 2105-2111.

Kojima, J., Y. Ikeda, and T. Nakajima (2000). Spatially resolved measurement of $\mathrm{OH}^{*}, \mathrm{CH}^{*}$, and $\mathrm{C} 2 *$ chemiluminescence in the reaction zone of laminar methane/air premixed flames. Proc. Combust. Inst. 28(2), $1757-1764$.

Kostiuk, L. W. and R. K. Cheng (1994). Imaging of premixed flames in microgravity. Exp. Fluids 18(1-2), 59-68.

Kostiuk, L. W. and R. K. Cheng (1995). The coupling of conical wrinkled laminar flames with gravity. Combust. Flame 103(1-2), 27-40.

Lauer, M. and T. Sattelmayer (2010). On the adequacy of chemiluminescence as a measure for heat release in turbulent flames with mixture gradients. J. Eng. Gas Turb. Power 132, 061502 (8 pages).

Lauer, M., M. Zellhuber, C. Aul, and T. Sattelmayer (2011). Determination of the Heat Release Distribution in Turbulent Flames by a Model Based Correction of $\mathrm{OH}^{*}$ Chemiluminescence. In Proceedings of the ASME Turbo Expo 2011, Number GT2011-45105.

Ledder, G. and A. Kapila (1991). The response of premixed flames to pressure perturbations. Combust. Sci. Tech. 76(1-3), 21-44. 
Li, J., F. Richecoeur, Y. Huang, and T. Schuller (2011). An acoustic methodology to measure heat release rate fluctuations from unsteady laminar flames. In Proceedings of the ASME Turbo Expo 2011, Number GT2011-46431 (10 pages).

Lieuwen, T. (2001). Theory of high frequency acoustic wave scattering by turbulent flames. Combust. Flame 126(1-2), 1489-1505.

Lieuwen, T., R. Rajaram, Y. Neumeier, and S. Nair (2002). Measurements of incoherent acoustic wave scattering from turbulent premixed flames. Proc. Combust. Inst. 29(2), 1809-1815.

Lieuwen, T. C. and V. Yang (Eds.) (2005). Combustion instabilities in gas turbines, Operational experience, Fundamental mechanisms, and Modeling, Volume 210 of Progress in Astronautics and Aeronautics. AIAA, Inc.

Linteris, G. and I. Rafferty (2008). Flame size, heat release, and smoke points in materials flammability. Fire Safety J. 43(6), 442-450.

Mahafza, B. R. and A. Z. Elsherbeni (2004). Matlab Simulations for Radar Systems Design. Chapman and Hall, New York.

McIntosh, A. C. (1991). Pressure disturbances of different length scales interacting with conventional flames. Combust. Sci. Tech. 75(4-6), 287309.

Merrill, S. (2008). Radar Handbook, 3rd Edition. McGraw-Hill, New York. Najm, H., P. Paul, C. Mueller, and P. Wyckoff (1998). On the adequacy of certain experimental observables as measurements of flame burning rate. Combust. Flame 113(3), 312-332. 
Nguyen, Q.-V. and P. H. Paul (1996). The time evolution of a vortex-flame interaction observed via planar imaging of ch and oh. Proc. Combust. Inst. $26(1), 357-364$.

Nori, V. N. and J. M. Seitzman (2009). CH* chemiluminescence modeling for combustion diagnostics. Proc. Combust. Inst. 32(1), 895-903.

Oljaca, M., A. Glezer, M. Baffico, and F. Lund (1998). Ultrasound scattering by a swriling jet. Phys. Fluids 10(4), 886-898.

Oppenheim, V. and R. Schafer (1975). Digital signal processing. PrenticeHall.

Palies, P., D. Durox, T. Schuller, and S. Candel (2010). The combined dynamics of swirler and turbulent premixed swirling flames. Combust. Flame 157(9), 1698-1717.

Paul, P. and H. Najm (1998). Planar laser-induced fluorescence imaging of flame heat release rate. Proc. Combust. Inst. 27(1), 43-50.

Peters, N. and G. Ludford (1983). The effect of pressure variations on premixed flames. Combust. Sci. Tech. 34(1-6), 331-344.

Poulain, C., N. Mazellier, P. Gervais, Y. Gagne, and C. Baudet (2004). Spectral vorticity and lagrangian velocity measurements in turbulent jets. Flow Turbul. Combust. 72(2-4), 245-271.

Price, R., I. Hurle, and T. Sugden (1969). Optical studies of the generation of noise in turbulent flames. Proc. Combust. Inst. 12(1), 1093-1102.

Richecoeur, F., S. Ducruix, P. Scouflaire, and S. Candel (2009). Effect of temperature fluctuations on high frequency acoustic coupling. Proc. 
Combust. Inst. 32(2), 1663-1670.

Sadanandan, R., M. Stöhr, and W. Meier (2008). Simultaneous OH-PLIF and PIV measurements in a gas turbine model combustor. Appl. Phys. B-Lasers Opt. 90(3-4), 609-618.

Samaniengo, J., F. Egolfopoulos, and C. Bowman (1995). CO2* chemiluminescence in premixed flames. Combust. Sci. and Tech. 109(1-6), $183-203$.

Schuller, T., D. Durox, and S. Candel (2002). Dynamics of and noise radiated by a perturbed impinging premixed jet flame. Combust. Flame 128(1-2), 88-110.

Stöhr, M., I. Boxx, C. Carter, and W. Meier (2011). Dynamics of lean blowout of a swirl-stabilized flame in a gas turbine model combustor. Proc. Combust. Inst. 33(2), 2953-2960.

Tanahashi, M., S. Taka, M. Shimura, and T. Miyauchi (2008). CH doublepulsed PLIF measurement in turbulent premixed flame. Exp. Fluids $45(2), 323-332$.

Vagelopoulos, C. M. and J. H. Frank (2005). An experimental and numerical study on the adequacy of ch as a flame marker in premixed methane flames. Proc. Combust. Inst. 30(1), 241-249.

Wangher, A., G. Searby, and J. Quinard (2008). Experimental investigation of the unsteady response of premixed flame fronts to acoustic pressure waves. Combust. Flame 154(1-2), 310-318. 


\section{List of Figures}

1 Schematic of the configuration investigated. A sound wave generated by an ultrasonic emitter propagates through ambient air, the burned gases formed around a flame before reaching a receiver. The distance between the emitter and receiver is $L$. Waves propagates through the burned gases over a distance $L_{f} . \ldots \ldots \ldots \ldots \ldots \ldots$

2 Control volume divided in two parts by the flame: unburned gases (volume $V_{u}$ and mass flowrate $\dot{m}_{u}$ ) and burned gases (volume $V_{b}$ and mass flowrate $\dot{m}_{b}$ ). . . . . . . . . 36

3 Schematic view of the experimental setup. . . . . . . . . . 37

4 Block diagram for the pulse compression generation and detection measurement chains. . . . . . . . . . . . . 38

5 Schematic of a train of chirp signals. $f_{i}$ : chirp initial frequency; $\tau$ : chirp duration; $t_{r}$ : repetition time; $B$ : chirp frequency bandwidth. . . . . . . . . . . . . . . . . 39

6 Examples of the ideal incident signal (a) and transmitted signal (b) together with their cross-correlation (c). . . . . . . . . 40

7 Example of chirp record in the absence of flow, (a) signal from the signal synthesizer output, (b) signal recorded by M1, (c) signal recorded by M2. . . . . . . . . . . . . . . . . . . 41

8 Pre-processing of signal measured by M1. (a) high pass filtered signal; (b) output of the multiplication between the filtered signal and the rectangular box function; (c) envelope of the signal; (d) normalized signal. . . . . . . . . . . . . . . . 42

9 Comparison of the cross-correlations with (black line) and without pre-processing (gray line). . . . . . . . . . . . 43

10 Effects of the chirp parameters on the determination of the sound travel time $\Delta t$. See Tab. 1 for values of the remaining parameters. The distance between the transmitter and receiver is $L=300 \mathrm{~mm}$. The speed of sound at ambient conditions is $c_{o}=348 \mathrm{~m} \cdot \mathrm{s}^{-1}$. Theoretically, the sound travel time is $\Delta t_{t h}=860 \mu \mathrm{s}$ in this case. . . . . . . . . . . 44 
11 Evolution of the sound travel time difference $\Delta\left(\Delta t_{0}\right)=\Delta t_{0}(L)-$ $\Delta t_{0}\left(L_{0}\right)$ with the distance $\Delta L=L-L_{0}$. The solid line indicates theoretical predictions deduced from Eq. (1). Markers denote measures in the absence of burner $(\square)$, with burner $(+)$, and for a non-reacting jet flow $(\star) . \ldots \ldots \ldots . . .45$

12 Color Schlieren images of a methane-air premixed flame $(\phi=$ $0.90, v_{u}=1.50 \mathrm{~m} \cdot \mathrm{s}^{-1}$ ) with and without quartz tube on the top of the flame. . . . . . . . . . . . . . . . . 46

13 Light emission (top) and sound propagation time (bottom) across a methane-air premixed flame $\left(\phi=0.90, v_{u}=1.50\right.$ $\mathrm{m} . \mathrm{s}^{-1}$ ) with and without flickering. . . . . . . . . . . . 47

14 Evolution of the mean flame emission intensity $\bar{I}$ as a function of the flow velocity $v_{u}$ for different equivalence ratios $\phi \ldots . .48$

15 Simultaneous records of chemiluminescence intensity perturbations $-I^{\prime}$ (solid line) and disturbances in the rate of change of the sound travel $d \Delta t / d t(+) \ldots \ldots \ldots$. . . . . . . . 49

16 Plot of the rate of change of travel time of ultrasonic waves $d \Delta t / d t$ versus light intensity fluctuations of the flames $I^{\prime}$. Flame C2: $\phi=0.85$ and $v_{u}=1.24 \mathrm{~m} \cdot \mathrm{s}^{-1}$. . . . . . . . 50

17 Comparison between measurements and predictions (solid line) for the coefficient $\Xi$ for two equivalence ratios $\phi=0.85(\nabla)$ and $\phi=1.0(\circ)$ as function of the flow velocity $v_{u} \ldots \ldots . .51$ 


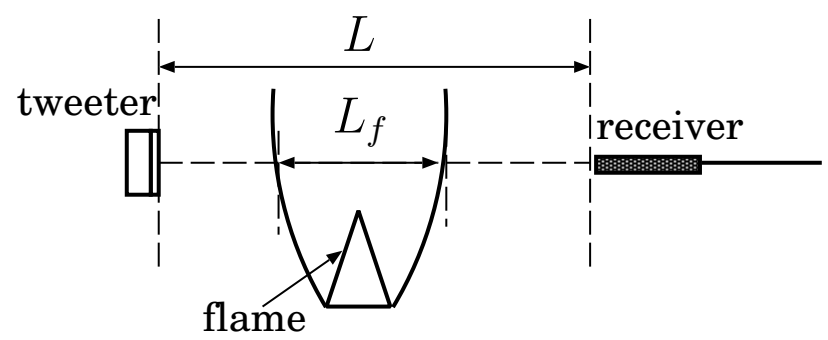

Figure 1: Schematic of the configuration investigated. A sound wave generated by an ultrasonic emitter propagates through ambient air, the burned gases formed around a flame before reaching a receiver. The distance between the emitter and receiver is $L$. Waves propagates through the burned gases over a distance $L_{f}$. 


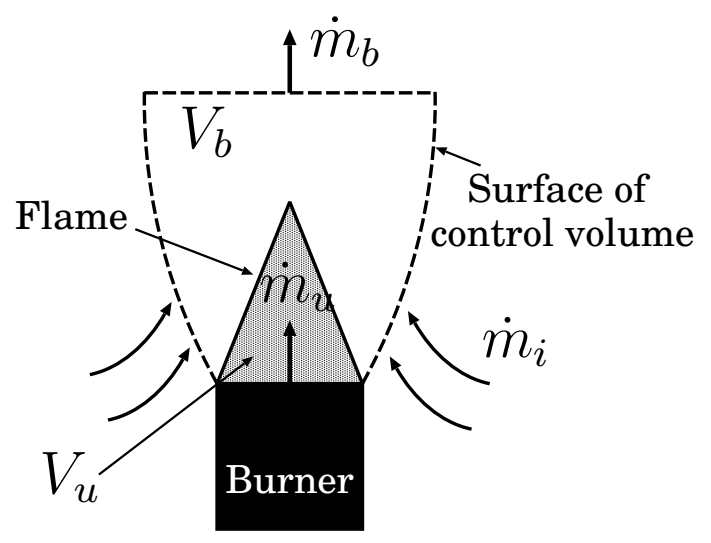

Figure 2: Control volume divided in two parts by the flame: unburned gases (volume $V_{u}$ and mass flowrate $\dot{m}_{u}$ ) and burned gases (volume $V_{b}$ and mass flowrate $\dot{m}_{b}$ ). 


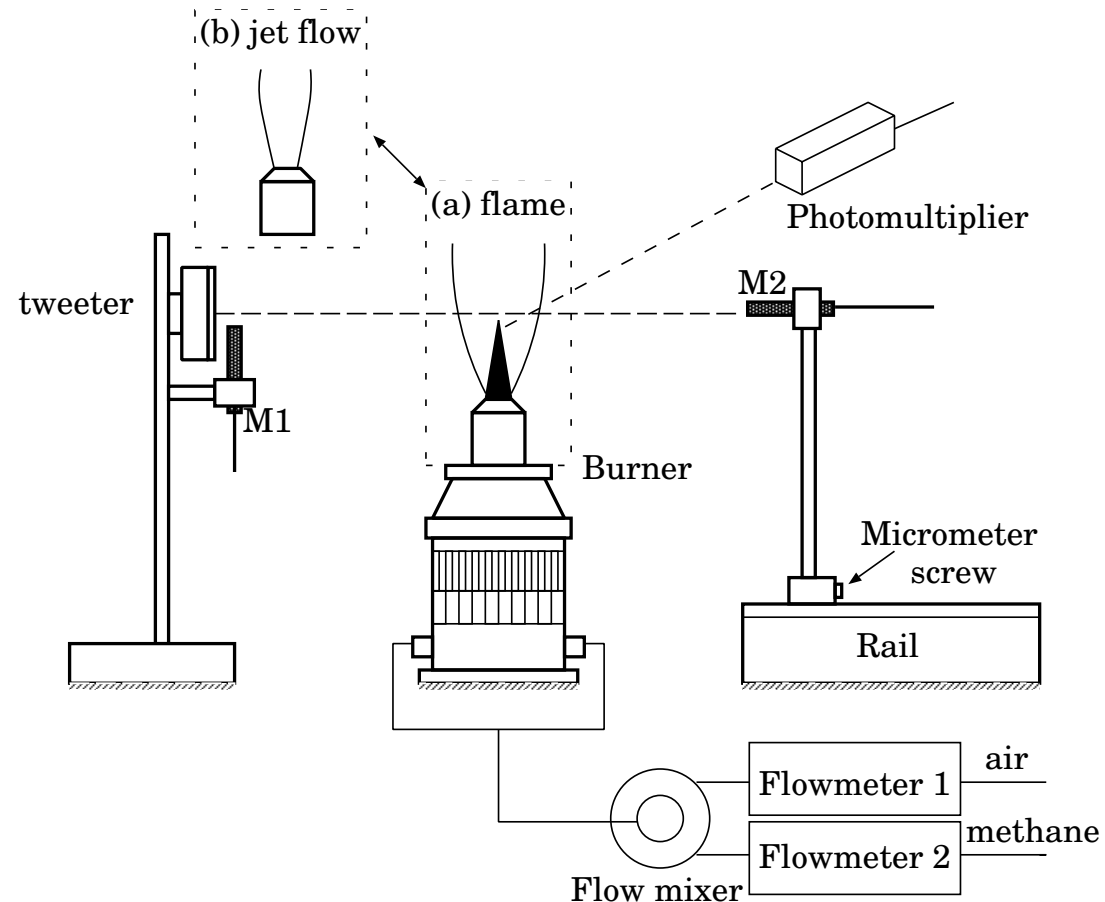

Figure 3: Schematic view of the experimental setup. 


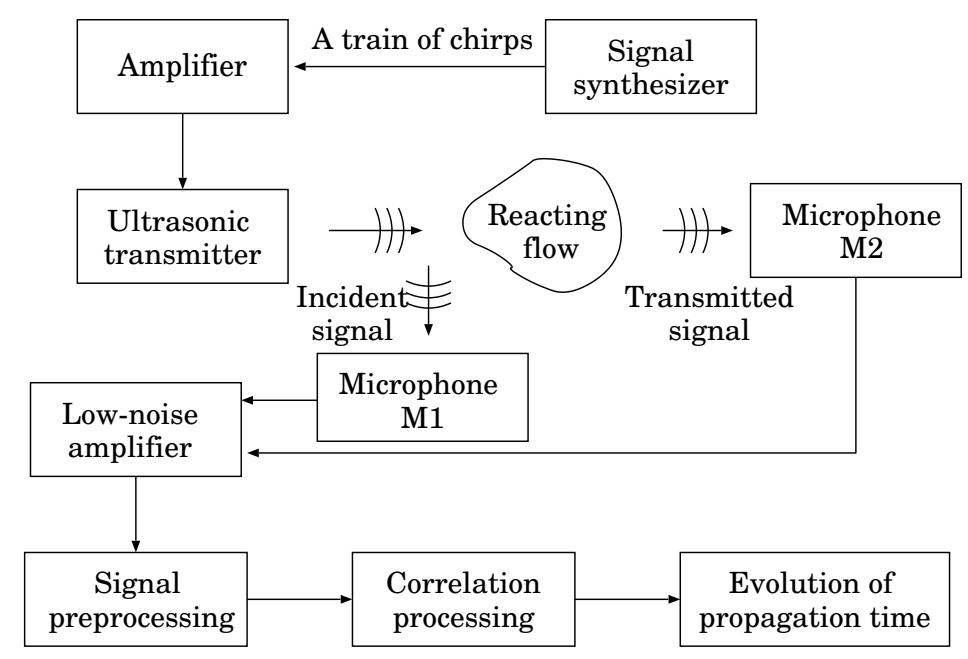

Figure 4: Block diagram for the pulse compression generation and detection measurement chains. 


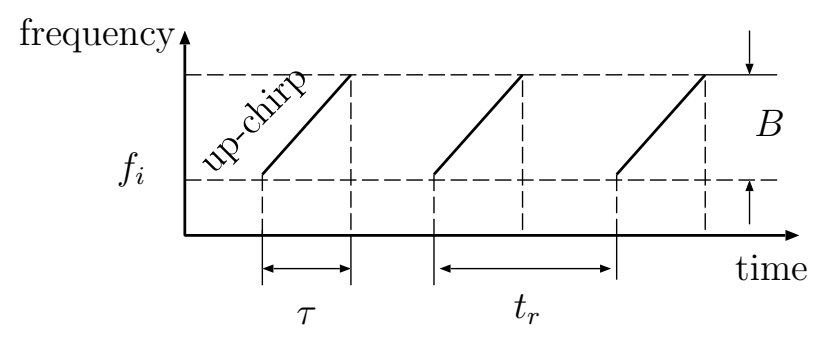

Figure 5: Schematic of a train of chirp signals. $f_{i}$ : chirp initial frequency; $\tau$ : chirp duration; $t_{r}$ : repetition time; $B$ : chirp frequency bandwidth. 


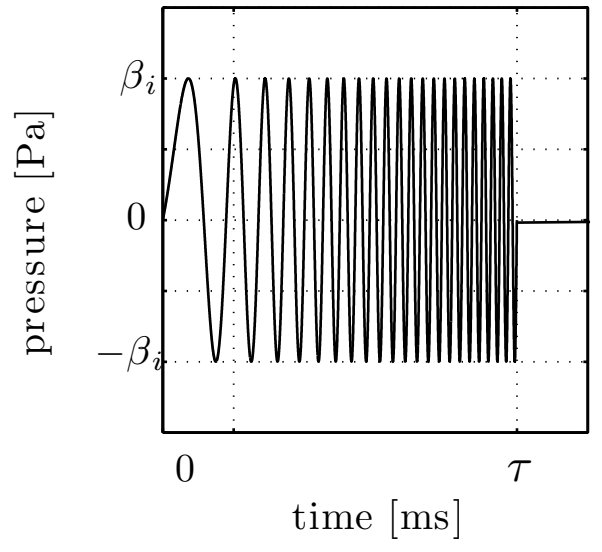

(a)

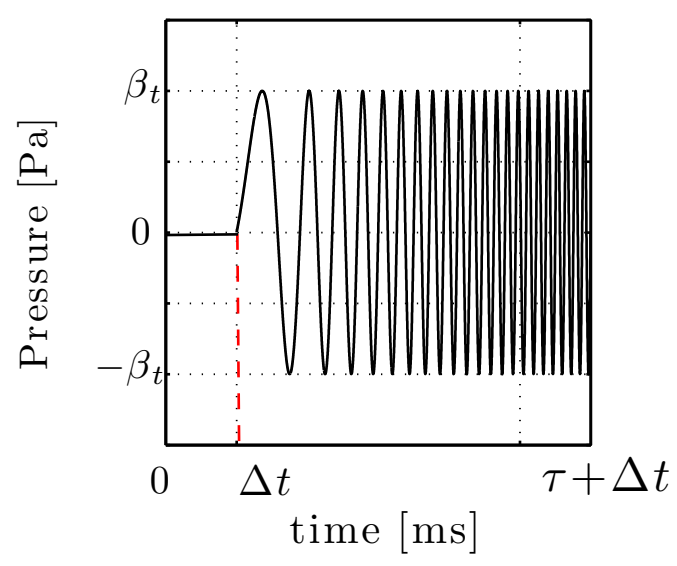

(b)

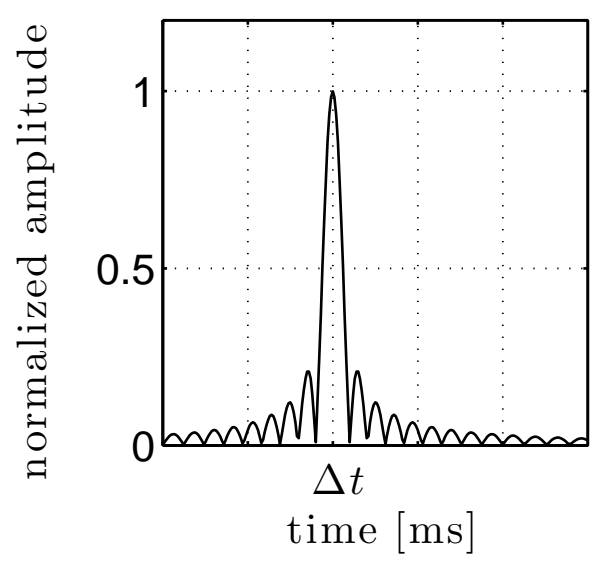

(c)

Figure 6: Examples of the ideal incident signal (a) and transmitted signal (b) together with their cross-correlation (c). 


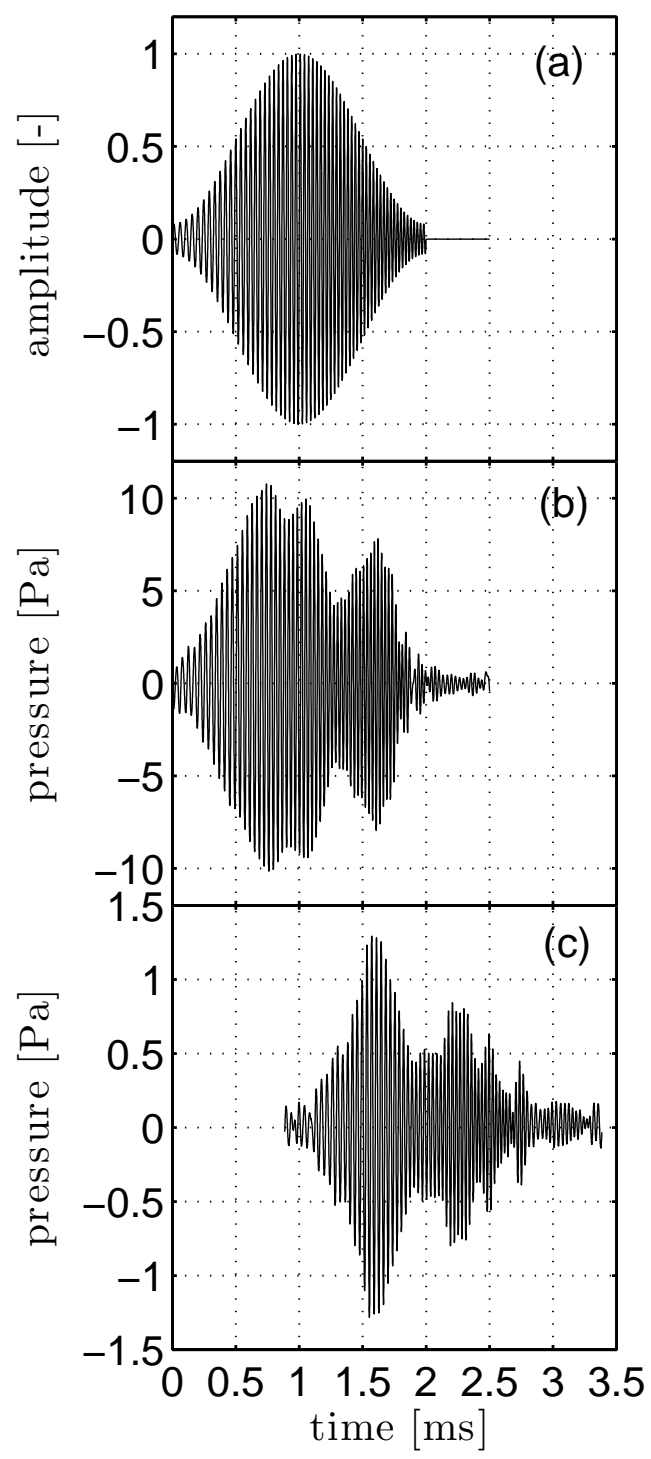

Figure 7: Example of chirp record in the absence of flow, (a) signal from the signal synthesizer output, (b) signal recorded by M1, (c) signal recorded by M2. 


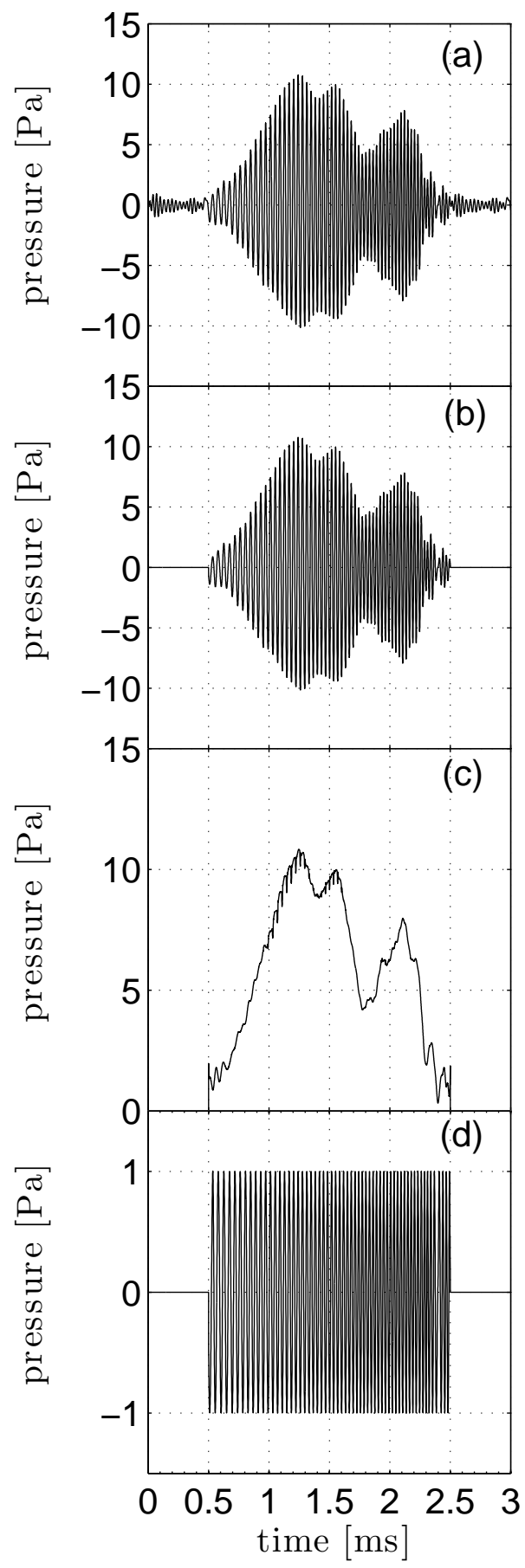

Figure 8: Pre-processing of signal measured by M1. (a) high pass filtered signal; (b) output of the multiplication between the filtered signal and the rectangular box function; (c) envelope of the signal; (d) normalized signal. 


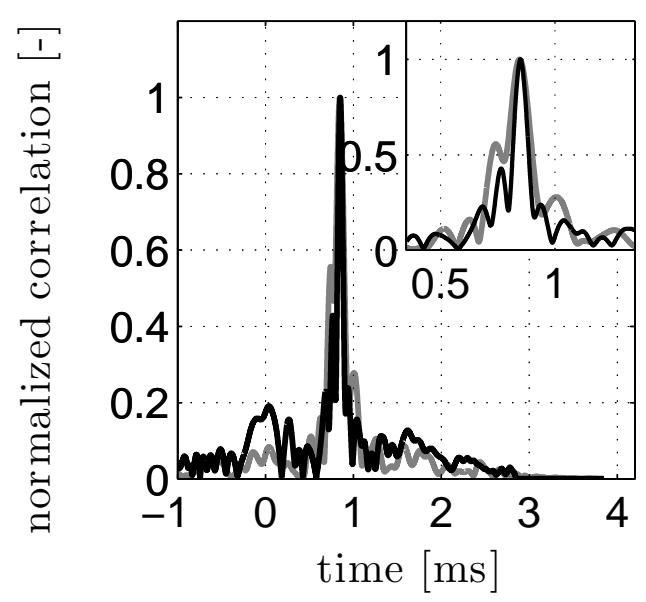

Figure 9: Comparison of the cross-correlations with (black line) and without pre-processing (gray line). 


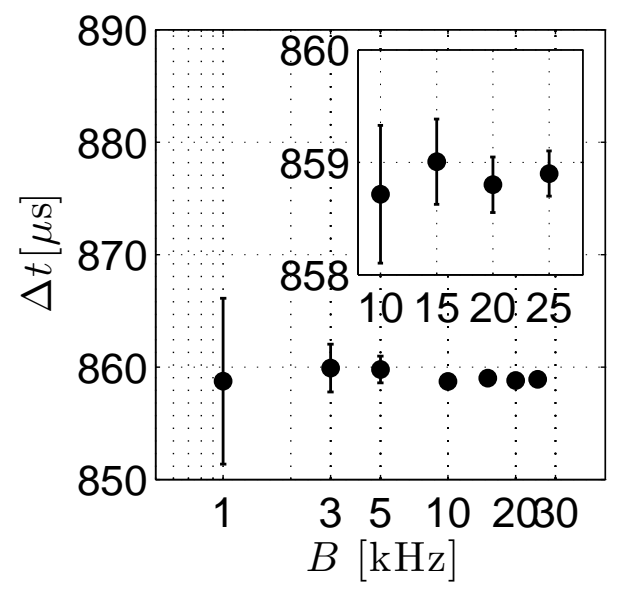

(a) Effect of $B$.

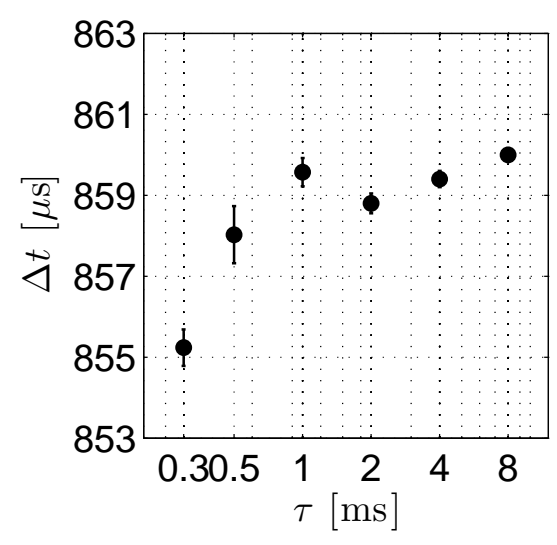

(b) Effect of $\tau$.

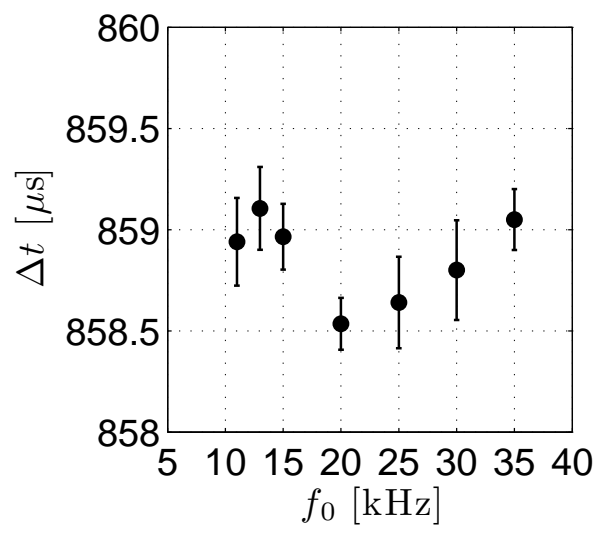

(c) Effect of $f_{0}$.

Figure 10: Effects of the chirp parameters on the determination of the sound travel time $\Delta t$. See Tab. 1 for values of the remaining parameters. The distance between the transmitter and receiver is $L=300 \mathrm{~mm}$. The speed of sound at ambient conditions is $c_{o}=348 \mathrm{~m} \cdot \mathrm{s}^{-1}$. Theoretically, the sound travel time is $\Delta t_{t h}=860 \mu$ s in this case. 


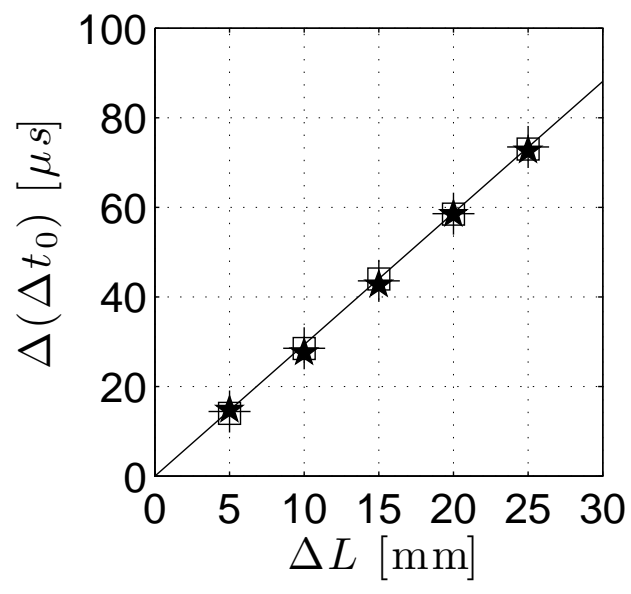

Figure 11: Evolution of the sound travel time difference $\Delta\left(\Delta t_{0}\right)=\Delta t_{0}(L)-\Delta t_{0}\left(L_{0}\right)$ with the distance $\Delta L=L-L_{0}$. The solid line indicates theoretical predictions deduced from Eq. (1). Markers denote measures in the absence of burner $(\square)$, with burner $(+)$, and for a non-reacting jet flow $(\star)$. 


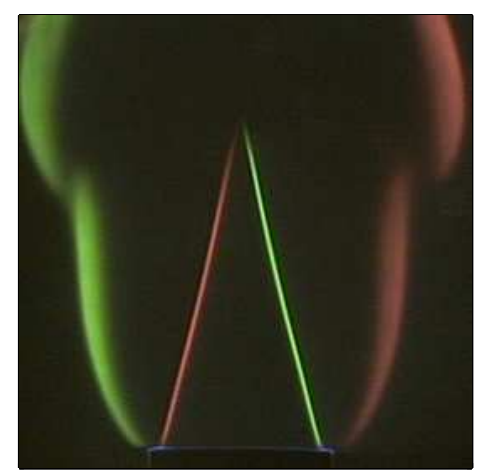

(a) Without quartz tube

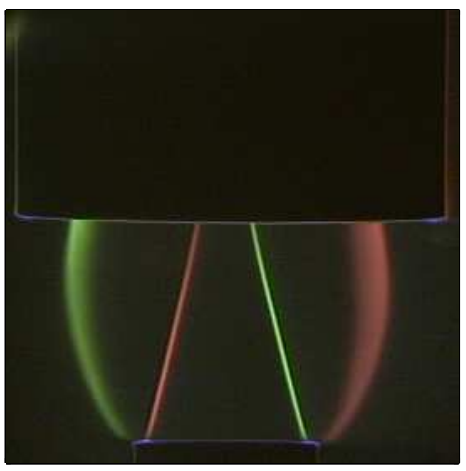

(b) With quartz tube

Figure 12: Color Schlieren images of a methane-air premixed flame $\left(\phi=0.90, v_{u}=1.50\right.$ $\mathrm{m} . \mathrm{s}^{-1}$ ) with and without quartz tube on the top of the flame. 


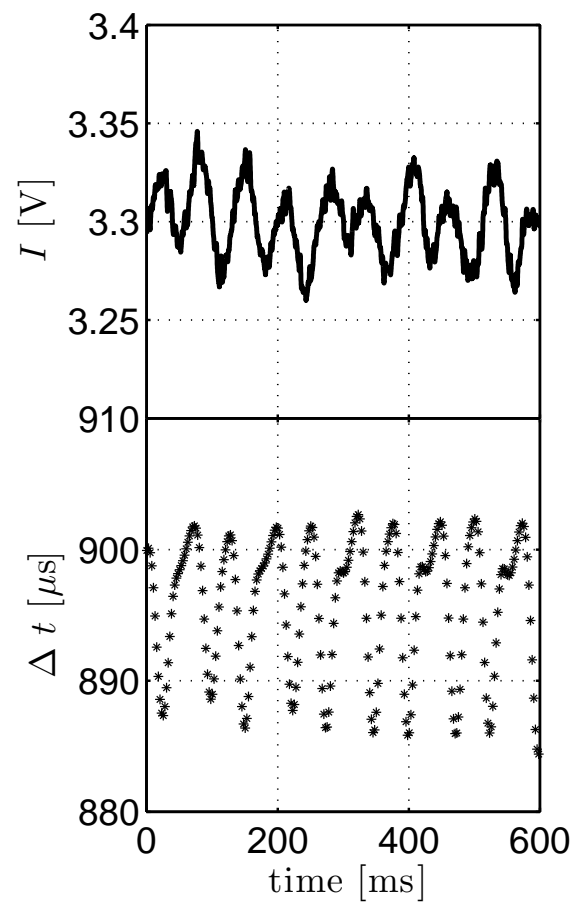

(a) With flickering

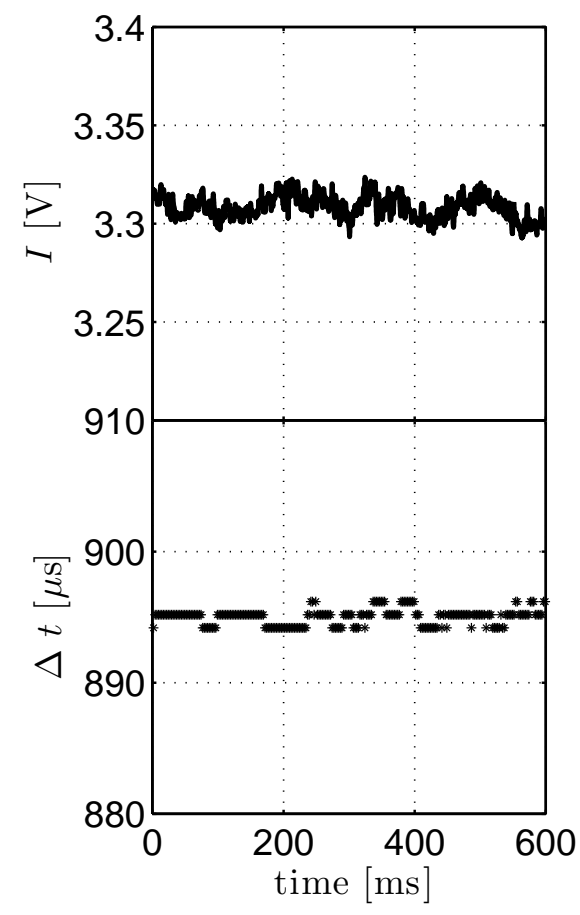

(b) Without flickering

Figure 13: Light emission (top) and sound propagation time (bottom) across a methane-air premixed flame $\left(\phi=0.90, v_{u}=1.50 \mathrm{~m} . \mathrm{s}^{-1}\right)$ with and without flickering. 


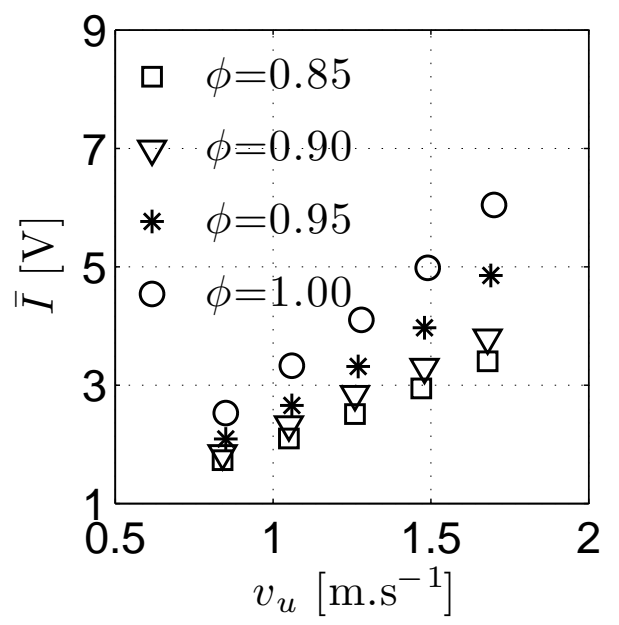

Figure 14: Evolution of the mean flame emission intensity $\bar{I}$ as a function of the flow velocity $v_{u}$ for different equivalence ratios $\phi$. 


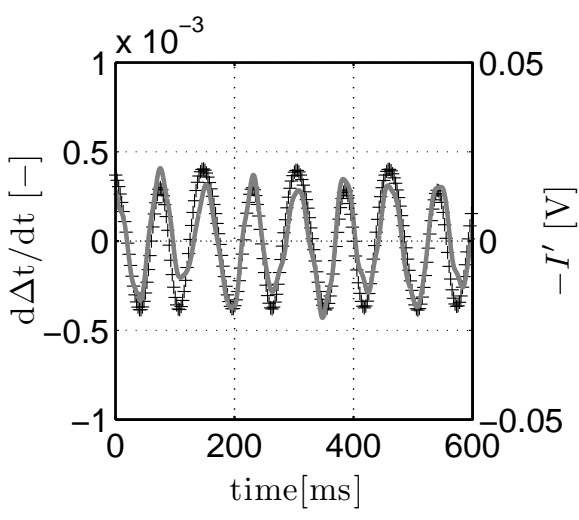

(a) $\mathrm{C} 1: \phi=0.85$ and $v_{u}=0.84 \mathrm{~m} . \mathrm{s}^{-1}$

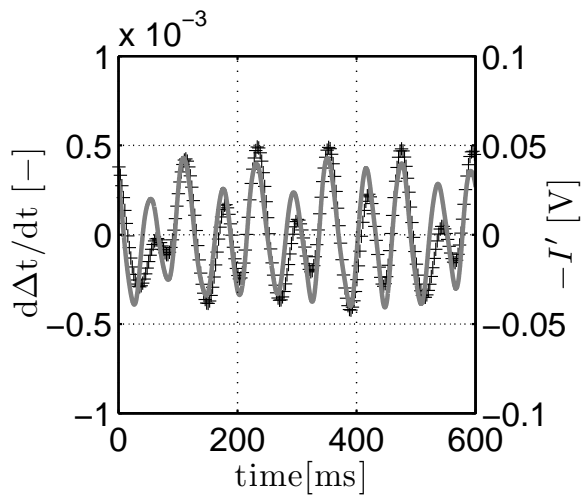

(c) C3: $\phi=0.85$ and $v_{u}=1.68 \mathrm{~m} . \mathrm{s}^{-1}$

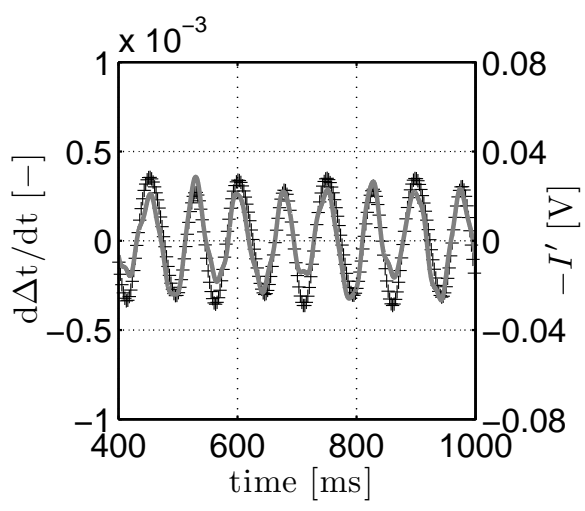

(e) C5: $\phi=0.95$ and $v_{u}=0.84 \mathrm{~m} . \mathrm{s}^{-1}$

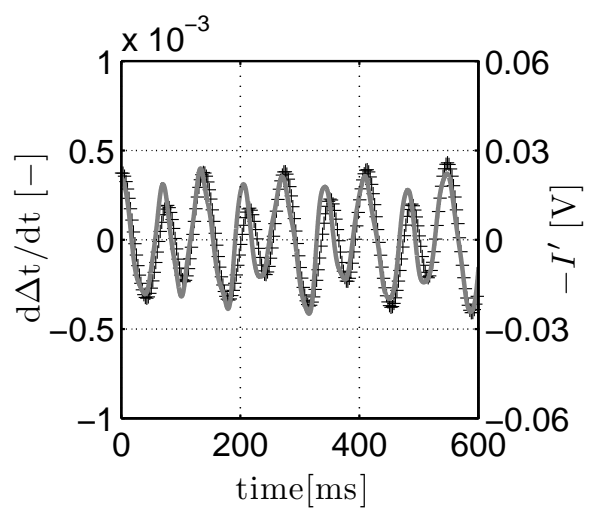

(b) $\mathrm{C} 2: \phi=0.85$ and $v_{u}=1.24 \mathrm{~m} . \mathrm{s}^{-1}$

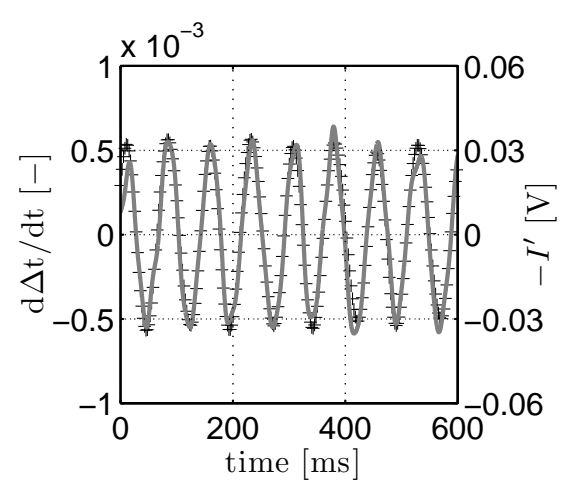

(d) C4: $\phi=0.90$ and $v_{u}=0.84 \mathrm{~m} . \mathrm{s}^{-1}$

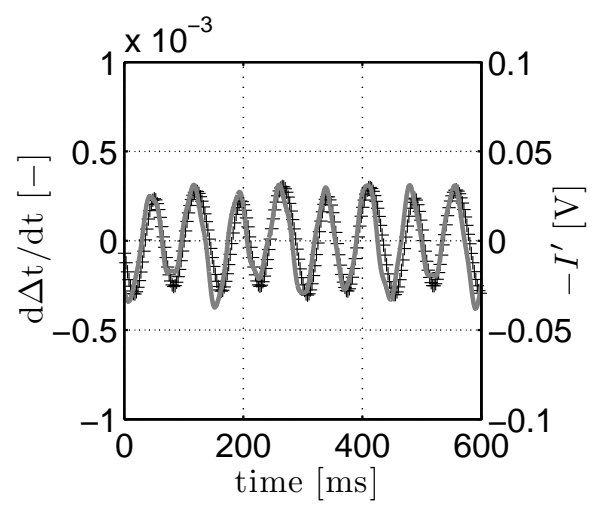

(f) C6: $\phi=1.00$ and $v_{u}=0.84 \mathrm{~m} . \mathrm{s}^{-1}$

Figure 15: Simultaneous records of chemiluminescence intensity perturbations $-I^{\prime}$ (solid line) and disturbances in the rate of change of the sound travel $d \Delta t / d t(+)$. 


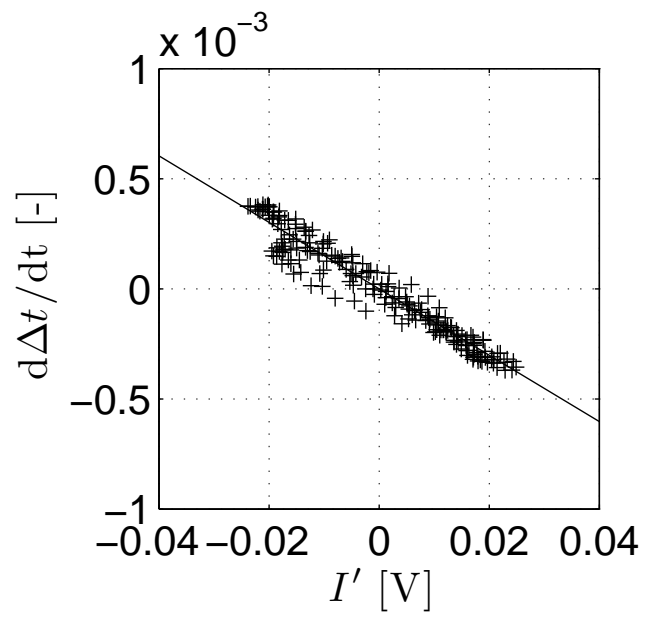

Figure 16: Plot of the rate of change of travel time of ultrasonic waves $d \Delta t / d t$ versus light intensity fluctuations of the flames $I^{\prime}$. Flame C2: $\phi=0.85$ and $v_{u}=1.24 \mathrm{~m} . \mathrm{s}^{-1}$. 


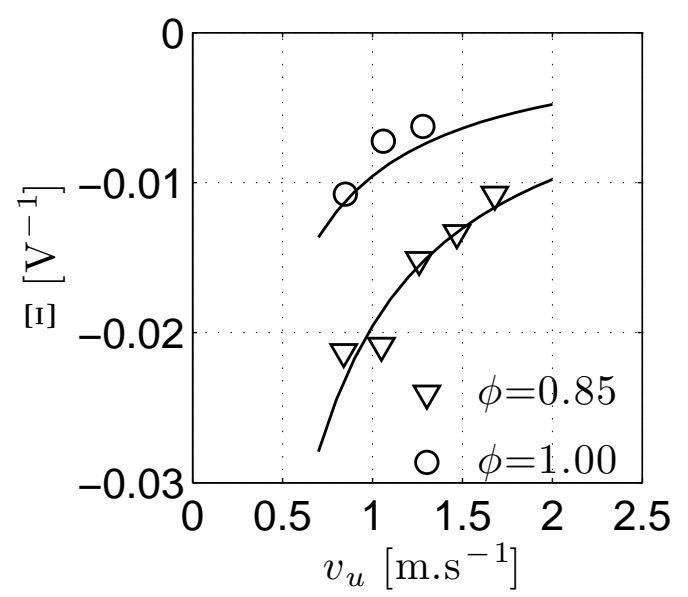

Figure 17: Comparison between measurements and predictions (solid line) for the coefficient $\Xi$ for two equivalence ratios $\phi=0.85(\nabla)$ and $\phi=1.0(\circ)$ as function of the flow velocity $v_{u}$. 
List of Tables

${ }_{667} 1$ Configurations tested for the chirp generation. . . . . . . . . 53

6682 Optimized values for the chirp parameters. . . . . . . . . . . 54

6693 Inlet flow velocity $v_{u}$ and equivalence ratio $\phi$ used for the six configurations explored $(\mathrm{Ci})$. The measured value of the coefficient $\Xi$ is also indicated together with the corresponding value of the linear regression factor $r^{2}$. . . . . . . . . 55 Numerical values used to estimate the coefficient $\Xi$ appearing in Eq. (16). . . . . . . . . . . . . . . . . . . 56 
Table 1: Configurations tested for the chirp generation.

\begin{tabular}{llll}
\hline Case & $\mathrm{B}[\mathrm{kHz}]$ & $\tau[\mathrm{ms}]$ & $f_{0}[\mathrm{kHz}]$ \\
\hline Effect of $B$ & $1-25$ & 2 & 30 \\
Effect of $\tau$ & 20 & $0.3-8$ & 30 \\
Effect of $f_{0}$ & 20 & 2 & $11-35$ \\
\hline
\end{tabular}


Table 2: Optimized values for the chirp parameters.

\begin{tabular}{llll}
\hline $\mathrm{B}[\mathrm{kHz}]$ & $\tau[\mathrm{ms}]$ & $f_{i}[\mathrm{kHz}]$ & $\tau_{r}[\mathrm{~ms}]$ \\
\hline 20 & 2 & 20 & 2.5 \\
\hline
\end{tabular}


Table 3: Inlet flow velocity $v_{u}$ and equivalence ratio $\phi$ used for the six configurations explored $(\mathrm{Ci})$. The measured value of the coefficient $\Xi$ is also indicated together with the corresponding value of the linear regression factor $r^{2}$.

\begin{tabular}{lcccccc}
\hline Case & C1 & C2 & C3 & C4 & C5 & C6 \\
\hline$\phi$ & 0.85 & 0.85 & 0.85 & 0.90 & 0.95 & 1.0 \\
$v_{u}\left[\mathrm{~m}^{-s^{-1}}\right]$ & 0.84 & 1.24 & 1.68 & 0.84 & 0.84 & 0.84 \\
$\Xi\left[\mathrm{V}^{-1}\right]$ & -0.0213 & -0.0151 & -0.0108 & -0.0137 & 0.0125 & -0.0107 \\
$r^{2}$ & 0.88 & 0.93 & 0.81 & 0.95 & 0.87 & 0.92 \\
\hline
\end{tabular}


Table 4: Numerical values used to estimate the coefficient $\Xi$ appearing in Eq. (16).

\begin{tabular}{ccccccc}
\hline$\phi$ & $S_{L}\left[\mathrm{~m} . \mathrm{s}^{-1}\right]$ & $c_{u}\left[\mathrm{~m} . \mathrm{s}^{-1}\right]$ & $c_{b}\left[{\left.\mathrm{~m} . \mathrm{s}^{-1}\right]}^{-1}\right.$ & $\Delta q\left[{\left.\mathrm{~kJ} . \mathrm{kg}^{-1}\right]}\right.$ & $\epsilon\left[\mathrm{V} . \mathrm{s} . \mathrm{m}^{-1}\right]$ & $\alpha$ \\
\hline 0.85 & 0.31 & 343 & 797 & 1740 & 1.99 & 0.1 \\
\hline 1.00 & 0.38 & 343 & 834 & 1930 & 4.08 & 0.1 \\
\hline
\end{tabular}

